\title{
ESTUDIOTRANSVERSAL DE LAS PRINCIPALES DIMENSIONES DE LA MANO EN ESTUDIANTES DE LA ESCUELA ACADÉMICO PROFESIONAL DE FARMACIA Y BIOQUÍMICA DE LA UNIVERSIDAD NACIONAL JORGE BASADRE GROHMANN - TACNA
}

\author{
CROSS - SECTONIAL STUDY OFTHE MIN DIMENSIONS OFTHE \\ HAND IN STUDETS ACADEMIC VOCATIONAL SCHOOL OF \\ PHARMACY AND BIOCHEMISTRY OFTHE NATIONAL UNIVERSITY \\ JORGE BASADRE GROHMANN - TACNA
}

\author{
Abelardo Emilio Rodríguez Menéndez'; Elfrén Raúl Liendo Liendo
}

RESUMEN

\begin{abstract}
Se trata del estudio transversal de las principales dimensiones de la mano en 19 jóvenes estudiantes de la Escuela Académico Profesional de Farmacia y Bioquímica, de la Facultad de Ciencias de la Salud - Universidad Nacional Jorge Basadre Grohmann - Tacna, comprendidos entre 18 y 20 años de edad, de ambos sexos. Mujer: Peso promedio:50 kg., talla promedio: 1.56; Hombre: Peso promedio: $72 \mathrm{~kg}$., talla promedio: 1.68 . Se midieron diez dimensiones de la mano que incluyen tres índices de longitud y dos de ancho, así como la longitud de los dedos. También se estudió la forma del dorso de la mano, dedos y uñas. Finalmente, se ofrecen datos de interés sobre el tipo de mano más frecuente y la fórmula de la longitud de los dedos en relación al sexo.
\end{abstract}

Palabras Clave: Mano tipo radial, mano tipo ulnar.

\section{ABSTRACT}

\begin{abstract}
We studied 19 young students from the academic vocational school of Pharmacy and Biochemistry from the Universidad Nacional Jorge Basadre Grohmann - Tacna, rangig between 18 ad 20 years of age. It is measured ten dimensions of the hand that includes three levels of length and the width and the length of the fingers. We study the shape of the back of the hand, fingers and nails. Finally we present interesting data on the most common type of hand and the formula for the length of the fingers in relation to sex.
\end{abstract}

Keywords: Hand radial, ulnar hand type.

\section{INTRODUCCIÓN}

Es una preocupación actual en el campo de la investigación morfológica, conocer las modificaciones que se producen en el organismo vivo del hombre como consecuencia de la acción, que sobre él, ejerce los factores internos y externos, reguladores del crecimiento y desarrollo.

Las investigaciones antropométricas en niños, jóvenes y adultos se encuentran en el primer plano de interés en el mundo. Muchos han sido los investigadores que han concentrado sus estudios sobre el crecimiento y el desarrollo de la mano[1][2][3].

Resulta de interés este primer estudio realizado, sobre las principales dimensiones de la mano en jóvenes estudiantes de la Escuela Académico Profesional de Farmacia y Bioquímica, de la Universidad Nacional Jorge Basadre GrohmannTacna, a fin de conocer el tipo más frecuente de mano, la fórmula de la longitud de los dedos y la forma del dorso de la mano, uñas y dedos.

Así como conocer si existen evidencias de lateralidad y diferencias en relación al sexo. El

\footnotetext{
Doctor en Medicina Humana, especialidad en Anatomía Humana en primer y segundo grado. Facultad de Ciencias de la Salud de la UNJBG.

${ }^{2}$ Magíster en Política Social con Mención en Salud y Seguridad Social. Facultad de Ciencias de la Salud de la UNJBG.
} 
estudio de tallado de la mano ofrece información en tal sentido y sirve para la evaluación de potencialidades físicas del individuo.

\section{OBJETIVOS}

Objetivo general: Conocer algunas características morfológicas de las manos en jóvenes peruanos.

Objetivos específicos: Conocer en relación al sexo el tipo de mano más frecuente, la forma del dorso de la mano, la forma de los dedos, la forma de las uñas y si existe diferencia entre las mediciones y observaciones realizadas en las manos de jóvenes Peruanos, con otras poblaciones.

\section{MATERIALES Y MÉTODOS}

La población está conformada por estudiantes del segundo año de la E.A.P. de Farmacia y Bioquímica, de la Facultad de Ciencias de la Salud de la Universidad Nacional Jorge Basadre Grohmann, Tacna. Doce son del sexo femenino y 7 , del sexo masculino, comprendidos entre 18 y 20 años de edad. El lado dominante se conoció preguntando con qué mano escribía y lanzaba la pelota. Resultaron ser diecisiete derechos y dos izquierdos. Se midieron diez dimensiones físicas en cada mano, que incluyen diez índices de longitud y dos de ancho, así como la longitud de los dedos, según describe Pospisil [4]

Además, se midió el peso y la talla. Las mediciones de la mano fueron realizadas con un glicier. Para la talla y el peso se utilizó una pesa fija, también se realizó un estudio cualitativo sobre la forma de los dedos y de los dorsos de las manos, formas de las uñas según escalas que reporta Pospisil [4].
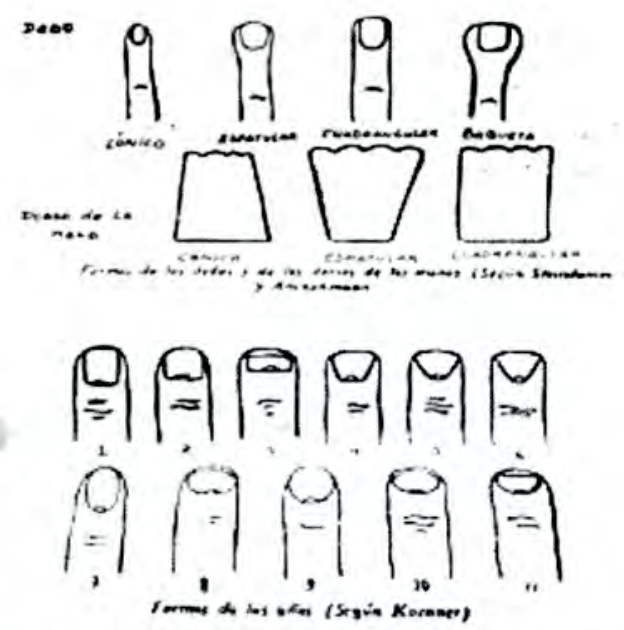

Gráfico $\mathrm{N}^{\circ}$ 01: Predominancia de la longitud de la mano.

Fuente: Propia
El análisis estadístico se realizó a través del test estadístico o paramétrico de Mann Witney, que es una alternativa de la " $t$ " de student.

\section{RESULTADOS Y DISCUSIÓN}

Las tablas I y II muestran la descripción estadística de las mediciones realizadas en las manos derecha e izquierda, en relación al sexo respectivamente. Se observó que en los índices de longitud de la mano el de mayor dimensión fue el índice medio siguiéndole en orden decreciente los índices ulnar y radial. En estudio similar realizado en Cuba en recién nacidos[5], se observó el predominio de la mano tipo radial.

La fórmula observada para la longitud de los dedos fue III - IV - II - V - I en ambas manos lo cual se corresponde con lo descrito por Testut y Latarjet [6] y Rodríguez y $\mathrm{Col}_{[}[7]$.

$\mathrm{Al}$ comparar las mediciones entre las manos derecha e izquierda en cada grupo no se observó evidencias de lateralidad en las variables estudiadas.

En las observaciones realizadas sobre el dorso de la mano se encontró que la forma más frecuente es la ESPATULAR, siguiéndole en frecuencia la cuadrangular y la cónica para ambos sexos. Según la clasificación de Steindamm y Anckermann que cita Pospisil [4].

En estudio similar realizado en Cuba en estudiantes universitarios de medicina humana, se observó que la forma más frecuente es la cuadrangular para el sexo masculino, mientras que en el sexo femenino fue la cónica[7].

$\mathrm{Al}$ analizar la forma de los dedos se encontró que en el sexo femenino las formas más frecuentes fueron la cónica y cuadrangular. En el estudio realizado en Cuba[7] se observa similares resultados. Mientras que en el sexo masculino fueron la espatular y la cónica, en el estudio realizado en Cuba[7] fueron la cuadrangular y la espatular.

Para el estudio de la forma de las uñas se utilizó la ESCALA DE KOENNER que cita Pospisil[1] y las clasifica en once tipos. Se observa que, en el sexo femenino, las formas más frecuentes son los tipos 7 y 3 , y en el sexo masculino, los tipos 1 y 2 .

En el estudio realizado en Cuba[7] los tipos de uñas son: el 7 para el sexo femenino y 7 y 11 para el masculino respectivamente.

Consideramos que las diferencias observadas en este estudio antropométrico detallado de la mano están condicionadas por las interacciones de 
factores hereditarios, del ambiente, sexuales, nutritivas y metabólicas.

\section{CONCLUSIONES}

- El tipo de mano más frecuente es el ulnar para ambos sexos.

- La fórmula encontrada para la longitud de los dedos son (III-IV-V-I)en ambos sexos.

- En las observaciones realizadas se encontró en el sexo femenino la forma ESPATULAR para el dorso de la mano y la cónica para la fórmula de los dedos con mayor frecuencia.

- Mientras que para el sexo masculino son la espatular para el dorso de la mano y la forma de los dedos respectivamente. Las formas de las uñas en el sexo femenino son los tipos 7 y 3 y para el sexo masculino los tipos 1 y 2 .

- No se encontró evidencias de lateralidad en las variables estudiadas.

\section{REFERENCIAS BIBLIOGRÁFICAS}

[1]POSPISIL, M. "Manual de la Practica de Antropologia Fisica". Cap. Métodos Generales en Antropología Física. Ed. Consejo Nacional de Universidades. La Habana, 1965, pp 1-11.

[2]MASHKARA, K; "Influence Phisical Activity in oldress process of some extremity bones" Arch. Anat. Gistal. Embriol. 69 (17); 1975 .
[3]YARBROUGH, C. "Stature and age as factors in the growth of second metacapol cortical bone in moderately mainoursched children” Ann Hum. Biol. 4(43); 1997.

[4]POSPISIL, M. "Manual de prácticas de antropología física" Cap. Somatología. Ed. Consejo Nacional de Universidades. La Habana 1965, pp 160-162.

[5]RODRÍGUEZ A. y COL; "Dimensiones Antropométricas de ambas manos en recién nacidos" II Jornada Científica de Ciencias Morfológicas. Cuba, 1982.

[6]TESTUT, L. Y LATARJET; "Anatomía Humana". Ed. P. Salvat, España, 1975

[7]RODRÍGUEZ, A. Y COL.; "Dimensiones Antropométricas de ambas manos en estudiantes de Medicina". II Jornada Científica de Ciencias Morfológicas. Cuba, 1982 .

\section{Correspondencia}

Abelardo Rodriguez Menéndez

Ciudad Universitaria fundo "Los Granados"

Av. Miraflores s/n Tacna - Perú

Elfrén Raúl Liendo Liendo

Ciudad Universitaria fundo "Los Granados"

Av. Miraflores s/n Tacna - Perú

\section{ANEXOS}

Tabla $\mathrm{N}^{\circ}$ 01: Resultados de las mediciones realizadas en la mano derecha $(\mathrm{en} \mathrm{cm})$.

\begin{tabular}{|c|c|c|c|}
\hline Mespictons & Fovianio & Masculno & SHOAIICATTO \\
\hline Inthoe modito & 16.83 & 17.74 & $\bullet$ \\
\hline folloe uhner & 15.04 & 1087 & - \\
\hline Golioe radilial & 1406 & 1697 & - \\
\hline Ancho do la mano & ont & 7.74 & - \\
\hline Ancho de la maiflecen & 47 & 807 & $\bullet$ \\
\hline Longlind del Primer dodo & 457 & S.S1 & $\bullet$ \\
\hline Longtiod del mogumdo dodo & 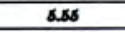 & as1 & $\bullet$ \\
\hline Longtiod del teroer dedo & 607 & 728 & $\bullet$ \\
\hline Longtiod del cuarto dodo & 800 & 687 & $\bullet$ \\
\hline Loagitud del quinto dodo & 40 & 800 & - \\
\hline
\end{tabular}

Fuente: Propia

Tabla $\mathrm{N}^{\circ}$ 02: Resultados de las mediciones realizadas en la mano izquierda $(\mathrm{en} \mathrm{cm})$.

\begin{tabular}{|c|c|c|c|}
\hline MEDICION & FMARnNo & MASCUInTO & SIGNIICATTVO \\
\hline Indice modio & 15.81 & 17.8 & - \\
\hline Indice uhar & 1497 & 160 & - \\
\hline Indioe rential & 1480 & 10.4 & $\bullet$ \\
\hline Ancho de he meno & 658 & 7.67 & - \\
\hline Ancho de ha mufect & $\mathbf{4 . 7}$ & 5.25 & - \\
\hline Longitud del primer dodo & $\mathbf{4 . 7 1}$ & 8.38 & - \\
\hline $\begin{array}{l}\text { Longitud del segundo } \\
\text { dedo }\end{array}$ & 8.51 & 28 & • \\
\hline Longitud del teroer dedo & as & 7.8 & - \\
\hline Longitud del cuarto dedo & 6 & 293 & - \\
\hline Longitud del quinto dodo & 4.57 & 5.34 & $\bullet$ \\
\hline
\end{tabular}

Fuente: Propia 